\title{
Scattering by an Arbitrary Cylinder at a Plane Interface: Broadside Incidence
}

\author{
EGON MARX
}

\begin{abstract}
The problem of the determination of the fields scattered by an infinite dielectric cylinder of arbitrary cross section located at the interface between two semi-infinite dielectric media is reduced to the solution of integral equations for unknown functions defined on the boundaries. These boundary functions are chosen so as to minimize their number. The incident field is that of a plane monochromatic wave. The derivation of the integral equations is given for the transverse electric (TE) mode for a dielectric cylinder and for a perfectly conducting cylinder. The exact electromagnetic fields are obtained from the solutions of the integral equations by integration, and the radar cross section can be computed from the far-field approximation. Sample outputs of the computer programs that implement this solution are shown.
\end{abstract}

\section{INTRODUCTION}

$\mathrm{R}$ ADAR CROSS SECTIONS have been determined for a large number of simple scatterers, and approximations are available when the linear size of the scatterer is either large or small compared to the wavelength. For arbitrary scatterers in the resonance region, where the size of the scatterer is comparable to the wavelength, the fields have to be computed by solving Maxwell's equations numerically, either directly or by first transforming them into integral equations.

We have reduced Maxwell's equations for the electromagnetic fields scattered by homogeneous obstacles to systems of integral equations for boundary functions in a way that the number of unknown functions is minimized, following methods developed for gratings [1], [2]. The electromagnetic fields are then obtained by integrating the solutions of these equations multiplied by the appropriate Green function over the boundary curves. We have used this approach for the scattering of transient and monochromatic waves by penetrable scatterers [3], specifically for electromagnetic scattering by a dielectric [4], and for transient scattering in dispersive media [5]. Glisson has shown [6] how the integral equations in [4] are derived using the equivalence principle. We have applied a stepping-in-time procedure to solve the integral equations for the scattering of transient fields by a threedimensional finite scatterer [7], using as a test case the scattering of a plane-wave pulse by a perfectly conducting sphere, a problem that can also be solved by means of a Mie power series expansion. The solution of a scattering problem for dielectrics and perfect conductors can be scaled to any region of the electromagnetic spectrum by preserving the ratio between the size of the scatterer and the wavelength or pulse

Manuscript received March 22, 1988: revised October 20. 1988.

The author is with the National Institute of Standards and Technology, Metrology Building, Room A117. Gaithersburg. MD 20899

IEEE Log Number 8927021. width; a finite conductivity has to be scaled inversely with the wavelength.

In this paper we show how to calculate the fields scattered by an infinite homogeneous dielectric or conducting cylinder of arbitrary cross section located at the plane interface between two semi-infinite media. A possible application is the determination of the radar cross section of a long object, for instance a ship, floating in water. We have also computed the fields scattered by a strip deposited on a substrate [8]; these fields are of interest in optical linewidth measurements for integratedcircuit devices.

We show how to compute the exact fields at any point and how to obtain the bistatic radar cross section from the far fields in either half-space. Although we assume that the interface outside the cylinder is a plane, the same equations are valid if this plane is deformed near the cylinder. This method can be generalized to arbitrary three-dimensional scatterers, with unknown functions defined on surfaces instead of curves.

We assume that the incident wave propagates through a medium with a real dielectric constant, but we allow the other two media to have complex dielectric constants to represent finite conductivity or other dissipative processes. We also derive the integral equations that apply when the cylinder is perfectly conducting. In [8] we derived the integral equations for scattering by an infinite strip of finite thickness for a number of combinations of dielectrics and conductors, and we have computed the near fields above a trapezoidal silver strip a fraction of a wavelength in height and in width on a glass substrate at optical frequencies [9], [10]. The problem of a strip located at the planar interface between two media is also discussed by Butler [11]. In this paper we restrict ourselves to broadside incidence: more specifically, we derive formulas for the transverse electric (TE) mode which can also be used with minor modifications for the transverse magnetic (TM) mode. In [12] we derive the equations for an incident wave of arbitrary polarization and direction of propagation, and we provide a more detailed presentation for some of the material in this paper.

In Section II we recall the solution of the Helmholtz equation by means of the Green function that satisfies the radiation condition at infinity. In Section III we define the boundary functions and derive the integral equations they satisfy for the TE mode. In Section IV we show how the radar cross section is computed from the far-field approximation of the field integrals. In Section $\mathrm{V}$ we discuss the numerical considerations that apply to the computer programs that implement these solutions, including possible difficulties with 


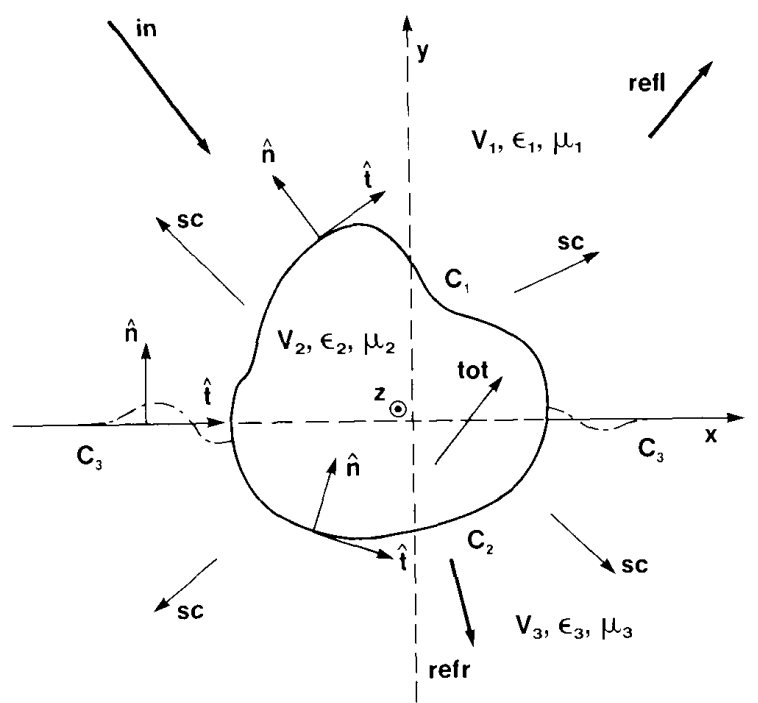

Fig. 1. Cross section of the cylinder showing the coordinate axes and the regions $V_{1}, V_{2}$, and $V_{3}$ separated by interfaces represented by the curves $C_{1}, C_{2}$, and $C_{3}$; the plane interface $C_{3}$ can be deformed near the cylinder. Also shown are the normals and tangents to the curves and the fields in the different regions.

infinite fields at sharp edges and spurious numerical instabilities in the integral equations at resonant frequencies of the interior problem, and we show graphic output obtained from computer programs that implement this method.

As shown in Fig. 1, we choose the $z$-axis in the plane interface and parallel to the generator of the cylinder, the $x$ axis also in this plane, and the $y$-axis pointing out of the substrate. Thus the plane interface is the $x z$-plane, and the wave vector of the incident wave is in the $x y$-plane for broadside incidence.

The contour of the cylinder is assumed to be partly over and partly under the boundary between the infinite media. The intersections of the interfaces and the $x y$-plane are the curves $C_{1}, C_{2}$, and $C_{3}$, and they can be represented by the parametric equations

$$
x=X_{i}(s), y=Y_{i}(s), i=1,2,3,
$$

where $s$ is the arclength. These equations allow for curves that may have more than one value of $y$ for a given $x$. The curve $C_{3}$ that corresponds to the interface between the two semi-infinite media is a straight line interrupted by the cylinder, and this line may be deformed near the cylinder. The definition of the unit normal to the curves shown in Fig. 1 is essentially arbitrary, and our choice comes from the problem in which the region $V_{2}$ extends to infinity as a layer in between the other regions [8].

When the incident wave is plane and monochromatic, the field has a periodicity in the $z$-direction that is maintained by the geometry and appears in all other fields. There is no $z$ dependence for broadside incidence.

\section{Solution of the Two-Dimensional Helmholtz. Equation}

We use the elementary solution or Green function to find the solution of the scalar Helmholtz equation

$$
\left(\nabla^{2}+k^{2}\right) U(x, y)=0
$$

in terms of the jumps of the function and of its normal derivative across an arbitrary curve $C$. In other words, we have to find a function $U$ that satisfies (2) in the whole $x y$ plane except on the curve $C$, that has a jump

$$
\Delta U=U_{+}-U_{-}=\phi
$$

across $C$, whose normal derivative has a jump

$$
\Delta\left(\frac{\partial U}{\partial n}\right)=\left(\frac{\partial U}{\partial n}\right)_{+}-\left(\frac{\partial U}{\partial n}\right)_{-}=\eta
$$

across $C$, and that satisfies the radiation condition everywhere at infinity. The choice of direction of the unit tangent $\hat{t}$, given by $\hat{t}=d \vec{X} / d s$, is shown in Fig. 1, and the unit normal $\hat{n}$ is then given by $\hat{n}=\hat{e}_{z} \times \hat{t}$. We designate by the subindices + and - the sides of the curve so that $\hat{n}$ points from - to + .

The Green function is proportional to the Hankel function of the first kind, $H_{0}^{(1)}$, and the solution $U$ can be written in terms of functionals of the jumps in the form

$$
U=G\{\eta\}+N\{\phi\},
$$

where

$$
\begin{gathered}
G\{\eta\}(\vec{x})=-\frac{i}{4} \int_{C} d s^{\prime} \eta\left(s^{\prime}\right) H_{0}^{(1)}(k R), \\
N\{\phi\}(\vec{x})=\frac{i}{4} \int_{C} d s^{\prime} \phi\left(s^{\prime}\right) H_{1}^{(1)}(k R) k \hat{n}^{\prime} \cdot \hat{R},
\end{gathered}
$$

$\vec{x}^{\prime}=\vec{X}\left(s^{\prime}\right), \vec{R}=\vec{x}-\vec{x}^{\prime}, R=|\vec{R}|, \hat{R}=\vec{R} / R$, and $\hat{n}^{\prime}=$ $\hat{n}\left(s^{\prime}\right)$. The functional notation in (6) and (7) means that, given a function $\eta$ or $\phi$ on $C$, we can find the value of $G\{\eta\}$ or $N\{\phi\}$ at any point $\vec{x}$ in the plane by evaluating the integral.

The limiting value of the function $U$ at either side of $C$ is

$$
U_{ \pm}= \pm \frac{\phi}{2}+\left.U\right|_{C}
$$

which satisfies the jump condition (3). We also see from (8) that the value of $U$ on $C$ is the average of the values on either side of $C$. If $\phi$ vanishes, that is, if $U$ is continuous across $C$, the limiting value of the normal derivative on either side of $C$ is

$$
\left(\frac{\partial U}{\partial n}\right)_{ \pm}= \pm \frac{\eta}{2}+\left(\frac{\partial U}{\partial n}\right)_{C} .
$$

Since the integrands in (6) and (7) become singular when the integrals are evaluated for a point on the curve $C$, the values of $U$ and $\partial U / \partial n$ on $C$ are defined by excluding a vanishingly small region around this point, as a kind of principal-value integral.

\section{Derivation of the Integral Equations}

In this section we derive the integral equations for the boundary functions and the integrals for the scattered fields.

We define auxiliary fields that equal the z-component of a physical field in one of the regions and satisfy other conditions that allow us to use the formal solutions derived in Section II. 
That is, these fields are defined in the $x y$-plane and they obey a Helmholtz equation with the same $k^{2}$ in the whole plane, satisfy the radiation condition everywhere at infinity, and are determined by jumps of the field and of its normal derivative at the boundary curves. We choose some of these jumps as the unknown boundary functions in the integral equations. We use the definitions of the auxiliary fields and the boundary conditions satisfied by the physical fields to express the auxiliary fields in terms of the boundary functions and to derive a set of integral equations. The scattered fields can then be obtained from the solutions of these equations by integration over the boundary curves.

In Section III-A we present the solution of the scattering problem for the TE mode and in Section III-B we extend the solution to the perfectly conducting cylinder. The solution for the TM mode can be obtained from that of the TE mode by interchanging the electric and magnetic fields with minor modifications. The case of arbitrary angle of incidence and polarization derived in [12] is not a superposition of TE and TM modes.

In the absence of the scatterer, the scattering of the plane monochromatic wave reduces to the well-known problem of reflection and refraction at a plane interface between two dielectrics. The incident, reflected, and refracted fields all obey the Maxwell equations without sources even on the boundaries. We subtract the incident and reflected fields from the total field in $V_{1}$ and the refracted from the total field in $V_{3}$ to define the scattered field in those regions. The reason we have to subtract the homogeneous fields to use the method presented here is that the solution (5) does not apply to total fields that do not satisfy the radiation condition at infinity. Since $V_{2}$ does not extend to infinity, we do not have to split the field there into parts. The different fields are also shown in Fig. 1.

The wave vectors $\vec{k}^{\prime}$ and $\vec{k}^{\prime \prime}$ and the amplitudes of the refracted field $E_{0 z}^{\prime}$ and the reflected field $E_{0 z}^{\prime \prime}$ are obtained from the continuity of the tangential components of the total fields $\vec{E}$ and $\vec{H}$. The homogeneous fields are assumed to be known when solving the integral equations.

The incident field is a plane monochromatic wave with the electric field parallel and the wave vector perpendicular to the $z$-axis.

The frequency of the fields is the same in the different media, whence the magnitudes of the wave vectors are related by

$$
k^{\prime 2} / \epsilon_{3} \mu_{3}=k^{2} / \epsilon_{1} \mu_{1}, k^{\prime \prime 2}=k^{2} .
$$

The wave vectors of the refracted and reflected fields then are

$$
\vec{k}^{\prime}=k_{x} \hat{e}_{x}-\left[\left(\mu_{3} \epsilon_{3} / \mu_{1} \epsilon_{1}\right) k^{2}-k_{x}^{2}\right]^{1 / 2} \hat{e}_{y}, \quad \vec{k}^{\prime \prime}=k_{x} \hat{e}_{x}-k_{y} \hat{e}_{y} .
$$

Since the components of the wave vector of the incident wave are real, only $k_{y}^{\prime}$ can be complex. A complex $k_{y}^{\prime}$ means that the refracted wave is attenuated exponentially with the distance from the interface, which happens when there are losses or in the case of total reflection.

To find the amplitudes of the refracted and reflected fields, we impose the continuity conditions for the tangential electric and magnetic fields and get

$$
\begin{gathered}
E_{0 z}+E_{0 z}^{\prime \prime}=E_{0 z}^{\prime}, \\
k_{y}\left(E_{0 z}-E_{0 z}^{\prime \prime}\right)=k_{y}^{\prime} E_{0 z}^{\prime},
\end{gathered}
$$

where we have set $\mu_{1}=\mu_{2}=\mu_{3}=\mu_{0}$. Once the $z$ components of the amplitudes of the reflected and refracted fields are determined, the fields on a curve $C_{i}$ are can be obtained by including exponential factors of the form $\exp (i \vec{k} \cdot \vec{x})$.

\section{A. Scattering in the TE Mode}

We define three auxiliary fields, $U_{1}, U_{2}$, and $U_{3}$, related to four unknown boundary functions, $\eta_{11}, \eta_{13}, \eta_{32}$, and $\eta_{33}$. We choose the field $U_{1}$ to be equal to $E_{z}^{\mathrm{sc}}$ in $V_{1}$, to satisfy the same Helmholtz equation as in medium 1 in $V_{2}$ and $V_{3}$, to be continuous everywhere, and to satisfy the radiation condition as $y \rightarrow-\infty ; U_{1}$ also satisfies the radiation condition as $y \rightarrow \infty$ because $E_{z}^{\mathrm{sc}}$ corresponds to a local perturbation of the homogeneous fields in $V_{1}$. The normal derivative of $U_{1}$ has a jump $\eta_{11}$ across $C_{1}$, a jump $\eta_{13}$ across $C_{3}$, and a zero jump across $C_{2}$. We choose the field $U_{2}$ to be equal to $E_{z}$ in $V_{2}$ and to vanish in $V_{1}$ and $V_{3}$. We choose the field $U_{3}$ to be equal to $E_{z}^{\mathrm{sc}}$ in $V_{3}$, to satisfy the same Helmholtz equation as in medium 3 in $V_{1}$ and $V_{2}$, to be continuous everywhere, and to satisfy the radiation condition as $y \rightarrow \infty ; U_{3}$ also satisfies the radiation condition as $y \rightarrow-\infty$ because $E_{z}^{\mathrm{sc}}$ corresponds to a local perturbation of the homogeneous field in $V_{3}$. The normal derivative of $U_{3}$ has a jump $\eta_{32}$ across $C_{2}$, a jump $\eta_{33}$ across $C_{3}$, and a zero jump across $C_{1}$. The ratios of the permeabilities are

$$
\alpha_{1}=\mu_{1} / \mu_{2}, \alpha_{2}=\mu_{2} / \mu_{3}, \alpha_{3}=\mu_{1} / \mu_{3} .
$$

The fields $U_{1}$ and $U_{3}$ are given in terms of the jumps of their normal derivatives by

$$
\begin{aligned}
& U_{1}=G_{11}\left\{\eta_{11}\right\}+G_{13}\left\{\eta_{13}\right\}, \\
& U_{3}=G_{32}\left\{\eta_{32}\right\}+G_{33}\left\{\eta_{33}\right\},
\end{aligned}
$$

where the first index on a functional $G_{i j}$ refers to the constants $\epsilon_{i}$ and $\mu_{i}$ that determine the magnitude of the wave vector and the second one refers to the curve $C_{j}$ over which the integration is carried out. The field $U_{2}$ can be expressed in terms of the jumps of the field and the normal derivative across $C_{1}$ and $C_{2}$. Up to the sign, these jumps are equal to the values of $U_{2}$ and $\partial U_{2} / \partial n$ at the boundary in $V_{2}$, since $U_{2}$ vanishes outside that region. The values at $C_{1}$ are determined from the continuity conditions of the tangential electric field $E_{z}$ and the tangential magnetic field $-(i / \mu \omega) \partial E_{z} / \partial n$ across $C_{1}$; we have

$$
\begin{aligned}
U_{2-} & =E_{z-}=E_{z+}=E_{z}^{\mathrm{in}}+E_{z}^{\mathrm{refl}}+U_{1} \\
& =E_{z}^{1}+G_{11}^{1}\left\{\eta_{11}\right\}+G_{13}^{1}\left\{\eta_{13}\right\},
\end{aligned}
$$

$$
\begin{aligned}
\left(\partial U_{2} / \partial n\right)_{-} & =\left(\partial E_{z} / \partial n\right)_{-}=\alpha_{1}\left(\partial E_{z} / \partial n\right)_{+} \\
& =\alpha_{1} \partial\left(E_{z}^{\text {in }}+E_{z}^{\mathrm{reff}}\right) / \partial n+\alpha_{1}\left(\partial U_{1} / \partial n\right)_{+} \\
& =\alpha_{1}\left(\partial E_{z}^{1} / \partial n+\frac{1}{2} \eta_{11}+N_{11}^{\prime 1}\left\{\eta_{11}\right\}+N_{13}^{\prime 1}\left\{\eta_{13}\right\}\right),
\end{aligned}
$$


where $E_{z}^{1}$ is the sum of the $z$-components of the incident and reflected fields evaluated on $C_{1}$, and the superindex on a functional indicates on which curve the field point $\vec{x}$ is located. The functional $N^{\prime}$ is the normal derivative of $G$ and differs only slightly from $N$; it is

$$
N^{\prime}\{\eta\}=\hat{n} \cdot \nabla G\{\eta\}=\frac{i}{4} \hat{n} \cdot \int_{C} d s^{\prime} \eta\left(s^{\prime}\right) H_{1}^{(1)}(k R) k \hat{R} .
$$

Note that $\hat{n}$ and consequently $N^{\prime}$ are defined only if $\vec{x}$ is on a specified curve, which may or may not be $C$ itself, while $N$ is defined for all $\vec{x}$. Similarly, the values of $U_{2}$ and $\partial U_{2} / \partial n$ at $C_{2}$ are

$$
\begin{aligned}
& U_{2+}=E_{z+}=E_{z-}=E_{z}^{\text {refr }}+U_{3} \\
& =E_{z}^{\mathrm{refr}}+G_{32}^{2}\left\{\eta_{32}\right\}+G_{33}^{2}\left\{\eta_{33}\right\}, \\
& \left(\partial U_{2} / \partial n\right)_{+}=\left(\partial E_{z} / \partial n\right)_{+}=\alpha_{2}^{-1}\left(\partial E_{z} / \partial n\right) \\
& =\alpha_{2}^{-1} \partial E_{z}^{\mathrm{refr}} / \partial n+\alpha_{2}^{-1}\left(\partial U_{3} / \partial n\right)_{-} \\
& =\alpha_{2}^{-1}\left(\partial E_{i}^{\mathrm{refr}} / \partial n-\frac{1}{2} \eta_{32}\right. \\
& \left.+N_{32}^{\prime 2}\left\{\eta_{32}\right\}+N_{33}^{\prime 2}\left\{\eta_{33}\right\}\right),
\end{aligned}
$$

where $E_{z}^{\text {refr }}$ is the $z$-component of the refracted field (here evaluated at $C_{2}$ ).

In (17), (18), (20), and (21) continuous fields such as $U_{1}$ and $U_{3}$ do not require an index + or - at the boundaries. We use these equations to express $U_{2}$ in terms of the jumps across $C_{1}$ and $C_{2}$, which in turn are functions of $\eta_{11}, \eta_{13}, \eta_{32}$, and $\eta_{33}$. The jumps of $U_{2}$ and of $\partial U_{2} / \partial n$ across $C_{1}$ and $C_{2}$ determine $U_{2}$ everywhere by means of (5), which takes the form

$$
\begin{aligned}
U_{2}=-G_{21}\left\{\left(\partial U_{2} / \partial n\right)_{-}\right. & \}-N_{21}\left\{U_{2-}\right\} \\
+ & G_{22}\left\{\left(\partial U_{2} / \partial n\right)_{+}\right\}+N_{22}\left\{U_{2+}\right\} .
\end{aligned}
$$

The integral equations for the boundary functions are obtained from the condition that $U_{2}$ vanish outside $V_{2}$ and the continuity conditions of the tangential components of the fields across $C_{3}$. To make $U_{2}$ vanish in $V_{1}$ and $V_{3}$ it suffices to set $U_{2}$ equal to zero just outside the boundaries $C_{1}$ and $C_{2}$ [3]; we obtain

$$
\begin{gathered}
U_{2+}=\frac{1}{2} \Delta U_{2}+\left.U_{2}\right|_{C_{1}}=-\frac{1}{2} U_{2}+\left.U_{2}\right|_{C_{1}}=0, \\
U_{2-}=-\frac{1}{2} \Delta U_{2}+\left.U_{2}\right|_{C_{2}}=-\frac{1}{2} U_{2+}+\left.U_{2}\right|_{C_{2}}=0 .
\end{gathered}
$$

After substitution from (17), (18), (20), (21), and (22), we find the integral equations

$$
\begin{gathered}
\left(\frac{1}{2} G_{11}^{1}+\frac{1}{2} \alpha_{1} G_{21}^{1}+\alpha_{1} G_{21}^{1} N_{11}^{\prime 1}+N_{21}^{1} G_{11}^{1}\right)\left\{\eta_{11}\right\} \\
+\left(\frac{1}{2} G_{13}^{1}+\alpha_{1} G_{21}^{1} N_{13}^{\prime 1}+N_{21}^{1} G_{13}^{1}\right)\left\{\eta_{13}\right\}
\end{gathered}
$$

$$
\begin{aligned}
& -\left(-\frac{1}{2} \alpha_{2}^{-1} G_{22}^{1}+\alpha_{2}^{-1} G_{22}^{1} N_{32}^{\prime 2}+N_{22}^{1} G_{32}^{2}\right)\left\{\eta_{32}\right\} \\
& -\left(\alpha_{2}^{-1} G_{22}^{1} N_{33}^{\prime 2}+N_{22}^{1} G_{33}^{2}\right)\left\{\eta_{33}\right\} \\
& =-\frac{1}{2} E_{z}^{1}-\alpha_{1} G_{21}^{1}\left\{\partial E_{z}^{1} / \partial n\right\}-N_{21}^{1}\left\{E_{z}^{1}\right\} \\
& +\alpha_{2}^{-1} G_{22}^{1}\left\{\partial E_{z}^{\mathrm{refr} / \partial n}\right\}+N_{22}^{1}\left\{E_{z}^{\mathrm{refr}}\right\}, \\
& -\left(\frac{1}{2} \alpha_{1} G_{21}^{2}+\alpha_{1} G_{21}^{2} N_{11}^{\prime 1}+N_{21}^{2} G_{11}^{1}\right)\left\{\eta_{11}\right\} \\
& -\left(\alpha_{1} G_{21}^{2} N_{13}^{\prime 1}+N_{21}^{2} G_{13}^{1}\right)\left\{\eta_{13}\right\} \\
& +\left(-\frac{1}{2} G_{32}^{2}-\frac{1}{2} \alpha_{2}^{-1} G_{22}^{2}+\alpha_{2}^{-1} G_{22}^{2} N_{32}^{\prime 2}+N_{22}^{2} G_{32}^{2}\right) \\
& \text { - }\left\{\eta_{32}\right\}+\left(-\frac{1}{2} G_{33}^{2}+\alpha_{2}^{-1} G_{22}^{2} N_{33}^{2}+N_{22}^{2} G_{33}^{2}\right)\left\{\eta_{33}\right\} \\
& =\frac{1}{2} E_{z}^{\mathrm{refr}}+\alpha_{1} G_{21}^{2}\left\{\partial E_{z}^{1} / \partial n\right\}+N_{21}^{2}\left\{E_{z}^{1}\right\} \\
& -\alpha_{2}^{-1} G_{22}^{2}\left\{\partial E_{z}^{\mathrm{refr}} / \partial n\right\}-N_{22}^{2}\left\{E_{z}^{\mathrm{refr}}\right\},
\end{aligned}
$$

where a composite functional such as $G N^{\prime}\{\eta\}$ stands for $G\left\{N^{\prime}\{\eta\}\right\}$. These composite functionals are aways defined because $G\{\eta\}$ and $N\{\phi\}$ are defined for all points in the $x y$ plane including those on the curves $C_{i}$ and $N^{\prime}\{\eta\}$ is defined on the $C_{i}$. We obtain two more integral equations from the continuity of the tangential electric field and the tangential component of the magnetic field on $C_{3}$. These equations are

$$
\begin{aligned}
& G_{11}^{3}\left\{\eta_{11}\right\}+G_{13}^{3}\left\{\eta_{13}\right\}-G_{32}^{3}\left\{\eta_{32}\right\}-G_{33}^{3}\left\{\eta_{33}\right\}=-E_{z}^{0} \\
& N_{11}^{\prime 3}\left\{\eta_{11}\right\}+\left(\frac{1}{2}+N_{13}^{\prime 3}\right)\left\{\eta_{13}\right\}-\alpha_{3} N_{32}^{\prime 3}\left\{\eta_{32}\right\} \\
& \quad+\alpha_{3}\left(\frac{1}{2}-N_{33}^{\prime 3}\right)\left\{\eta_{33}\right\}=-\partial \bar{E}_{z}^{0} / \partial n
\end{aligned}
$$

where

$$
E_{z}^{0}=E_{z}^{\text {in }}+E_{z}^{\text {refl }}-E_{z}^{\text {refr }}, \bar{E}_{z}^{0}=E_{z}^{\text {in }}+E_{z}^{\text {refl }}-\alpha_{3} E_{z}^{\text {refr }} .
$$

When the curves $C_{1}, C_{2}$, and $C_{3}$ are covered with patches, (25), (26), (27), and (28) provide the right number of linear algebraic equations for the unknown values of $\eta_{11}, \eta_{13}, \eta_{32}$, and $\eta_{33}$ at the centers of these patches. The functionals on the left side of the integral equations (25)-(28) do not depend on the angle of incidence, but the fields on the right side do.

If the interface remains unchanged outside the scatterer, the curve $C_{3}$ is a straight line and some simplifications are possible. The normal to $C_{3}$ is perpendicular to the vectors $\vec{R}$ that go from one point on this line to another, whence we can set

$$
N_{13}^{3}=N_{33}^{3}=0
$$

in (28). The boundary conditions for the homogeneous fields imply that

$$
E_{z}^{0}=0, \partial \bar{E}_{z}^{0} / \partial n=0
$$


in the $x y$-plane. For the case of a strip on a substrate, the curve $C_{2}$ continues the straight line of $C_{3}$ and further such simplifications occur.

The Poynting vector is expressed in terms of $E_{z}$ by

$$
\vec{S}=\frac{1}{2} \operatorname{Re}\left(\frac{i}{\omega \mu} E_{z} \nabla E_{z}^{*}\right) .
$$

We substitute $E_{z}$ from (15) and (16) to obtain the average energy flux of the scattered field in region $V_{1}$ and $V_{3}$, respectively. We can use this expression to find the energy flux at a given finite distance from the plane. Although the incident and reflected waves are assumed to be defined in all space and for all time, in practice they are confined to beams that last a finite time. If there is no interference between the scattered and the homogeneous fields, the vertical component of the Poynting vector in the region $V_{1}$ is given by

$$
\begin{aligned}
S_{y}(x, y)= & \frac{1}{32 \omega \mu_{1}} \operatorname{Im}\left\{\left[\int_{C_{1}} d s^{\prime} \eta_{11}\left(s^{\prime}\right) H_{0}^{(1)}(k R)\right.\right. \\
& \left.+\int_{C_{3}} d s^{\prime} \eta_{13}\left(s^{\prime}\right) H_{0}^{(1)}(k R)\right] \\
& \cdot\left[\int_{C_{1}} d s^{\prime} \eta_{11}^{*}\left(s^{\prime}\right) H_{1}^{(1) *}(k R) k\left(y-y^{\prime}\right) / R\right. \\
& \left.\left.+\int_{C_{3}} d s^{\prime} \eta_{13}^{*}\left(s^{\prime}\right) H_{1}^{(1) *}(k R) k\left(y-y^{\prime}\right) / R\right]\right\} .
\end{aligned}
$$

If we are in a region so close to the scatterer that the incident and reflected fields have to be taken into account, the energy flux must be computed by using the $z$-component of the total field in (32). A similar expression is valid for the energy flux of the scattered field in $V_{3}$, where we may have to take into account the refracted field.

\section{B. Scattering by a Perfectly Conducting Cylinder}

We again restrict ourselves to the TE mode. The fields inside the cylinder vanish, so we do not need the auxiliary field $U_{2}$. The remaining auxiliary fields defined in Section III-A are still related to the boundary functions $\eta_{i j}$ by (15) and (16). The boundary conditions change because we now allow for surface current and charge densities on the perfect conductor; the tangential component of the electric field and the normal component of the magnetic field are continuous, whence these components also have to vanish outside $V_{2}$ at $C_{1}$ and $C_{2}$. If $E_{z}$ vanishes on a curve, the tangential derivative $\partial E_{z} / \partial s$ also vanishes and thus the normal component of the magnetic field vanishes too. The remaining boundary conditions are

$$
E_{z+}=E_{z}^{\text {in }}+E_{z}^{\text {refl }}+U_{1}=E_{z}^{1}+G_{11}^{1}\left\{\eta_{11}\right\}+G_{13}^{1}\left\{\eta_{13}\right\}=0
$$

on $C_{1}$, and

$$
E_{z-}=E_{z}^{\mathrm{refr}}+U_{3}=E_{z}^{\mathrm{refr}}+G_{32}^{2}\left\{\eta_{32}\right\}+G_{33}^{2}\left\{\eta_{33}\right\}=0
$$

on $C_{2}$. These two integral equations and the equations obtained from the continuity of the tangential components of the fields across $C_{3}$, that is, (27) and (28), form a set of equations that determine the boundary functions. Note that $E_{*}$ and $\partial E_{z} / \partial s$ do not have to vanish on $C_{3}$.

\section{Radar Cross Sections}

The bistatic radar cross section of a scatterer in a medium such as air is given by [13], [14]

$$
\sigma(\hat{r})=\lim _{r \rightarrow \infty} 4 \pi r^{2}\left|\vec{E}^{\aleph}\right|^{2} /\left|\vec{E}^{\text {in }}\right|^{2},
$$

which is the ratio of the scattered to incident energy flux density adjusted for the $1 / r$ dependence of the far fields. This cross section in general depends on the direction of propagation of the incoming wave given by $\vec{k}$ and on the direction $\hat{r}(\theta, \phi)$ from the scatterer to the receiver. (In this section, $\theta$ and $\phi$ are the polar and azimuthal angles of the spherical coordinates, that is, $\phi$ is no longer the jump of a boundary function.) In the two-dimensional case the far fields are of the order of $1 / \sqrt{\rho}$, so the scattering cross section for broadside incidence is [14]

$$
\sigma(\phi)=\lim _{\rho \rightarrow \infty} 2 \pi \rho\left|\vec{E}^{\mathrm{sc}}\right|^{2} /\left|\vec{E}^{\text {in }}\right|^{2} .
$$

If medium 3 is conducting, the far fields in $V_{3}$ show an exponential decay with the distance from the interfaces and the limiting value in (37) vanishes. Therefore, we define the radar cross section in $V_{3}$ only if $\epsilon_{3}$ is real.

The far fields are obtained by using the large-argument approximation for the Hankel function $H_{0}^{(1)}$ in the functional $G$ in the expressions that allow us to calculate the scattered fields in both regions,

$$
H_{0}^{(1)}(\zeta) \approx(\pi \zeta / 2)^{-1 / 2} \exp [i(\zeta-\pi / 4)] .
$$

We expand $R$ in polar coordinates,

$$
R \approx \rho-\rho^{\prime} \cos \left(\phi-\phi^{\prime}\right),
$$

and obtain the far-field approximation

$$
\begin{aligned}
& G\{\eta\} \approx \exp [i(k \rho-3 \pi / 4)](8 \pi k \rho)^{-1 / 2} \\
& \cdot \int_{C} d s^{\prime} \eta\left(s^{\prime}\right) \exp \left[-i k \rho^{\prime} \cos \left(\phi-\phi^{\prime}\right)\right],
\end{aligned}
$$

where, in terms of the Cartesian coordinates (1) of $C$.

$$
\rho^{\prime} \cos \left(\phi-\phi^{\prime}\right)=X\left(s^{\prime}\right) \cos \phi+Y\left(s^{\prime}\right) \sin \phi .
$$

Then only the first factor on the right side of (40) contributes to the gradient term, so that we may write symbolically that $\nabla$ $\approx i k \hat{\rho}$ when the gradient is applied to a field, and minus that if it is applied to the complex conjugate of a field.

The intensity of the scattered electric field is obtained from (15) in $V_{1}$ and from (16) in $V_{3}$. The radar cross section (36) in $V_{1}$ becomes, for unit intensity of the incident electrical field,

$$
\begin{aligned}
\sigma_{1}(\phi)= & \frac{1}{4 k} \mid\left(\int_{C_{1}} d s^{\prime} \eta_{11}\left(s^{\prime}\right)+\int_{C_{3}} d s^{\prime} \eta_{13}\left(s^{\prime}\right)\right) \\
& \left.\cdot \exp \left\{-i k\left[X\left(s^{\prime}\right) \cos \phi+Y\left(s^{\prime}\right) \sin \phi\right]\right\}\right|^{2},
\end{aligned}
$$

with a similar expression for $\sigma_{3}(\phi)$ in $V_{3}$. The radiation pattern 
for a symmetric scatterer is symmetric, that is, $\sigma(\pi-\phi)=$ $\sigma(\phi)$, if $\eta(-X, Y)=\eta(X, Y)$, a condition that is satisfied for incidence normal to the plane.

\section{Numerical Considerations}

Here we indicate some of the approximations that are introduced to compute the boundary functions by solving the integral equations and the electromagnetic fields by integrating the solutions thus obtained. In Section V-A we show how the integral equations are transformed into a system of linear algebraic equations by the point-matching method. In Section $\mathrm{V}-\mathrm{B}$ we examine the computation of the fields near the cylinder, where we have to take into account the singularities of the Hankel functions.

For perfect conductors there are resonant modes of the interior problem that have no physical effect on the exterior problem, but that disturb the numerical computations and give rise to instabilities in the solution of the exterior problem [15], and similar problems occur for dielectric scatterers [16]. This is the subject of Section V-C. Another difficulty with numerical calculations is related to the field components that diverge at a sharp edge [17]-[20], and we address this problem in Section V-D.

We have written a group of computer programs to solve the problems of scattering by strips and cylinders of different composition and configurations. We show the results of numerical calculations in Section V-E.

\section{A. Point Matching}

To solve the system of weakly singular integral equations for the boundary functions we reduce the integral equations to a system of linear algebraic equations. We do this by covering the curves $C_{1}, C_{2}$, and $C_{3}$ with patches and by choosing the values of the boundary functions at the centers $\vec{x}_{l}=\vec{X}\left(s_{l}\right)$ of these patches as a discrete set of unknowns $\eta_{l}=\eta\left(s_{l}\right)$. The sizes of these patches do not have to be equal, and we cover only that part of the curve $C_{3}$, usually a few wavelengths away from the cylinder, where the values of the unknowns differ significantly from zero.

We have to approximate the linear functionals $G, N$, and $N^{\prime}$ by linear combinations of the unknowns. For instance, the value of the functional $G\{\eta\}$ at one of the points $\vec{x}_{l}$ becomes

$$
G\{\eta\}\left(\vec{x}_{l}\right) \approx \sum_{m} G_{l m} \eta_{m} .
$$

We approximate the integral in (6) by assuming that the integrand is constant over each patch; we obtain

$$
G_{l m}=-\frac{i}{4} \Delta s_{m} H_{0}^{(1)}\left(k R_{l m}\right), \quad l \neq m,
$$

where $\Delta s_{m}$ is the arclength of the patch $m$ and $R_{l m}=\mid \vec{x}_{l}-$ $\vec{x}_{m} \mid$. The contribution of the self-patch, where the field point and the source point coincide making the integrand diverge, can often be neglected. We compute an approximate value of this contribution by using the small-argument approximation of the Hankel function in the integrand and obtain

$$
G_{l l}=\frac{1}{2 \pi}\left[\log \left(\frac{1}{2} k \Delta s_{l}\right)-1.115931\right] \Delta s_{l}-\frac{i}{4} \Delta s_{l} .
$$

Similarly, we approximate $N$ and $N^{\prime}$ with sums of the form (43) and find

$$
N_{l m}=\frac{i}{4} \Delta s_{m} H_{1}^{(1)}\left(k R_{l m}\right) k \hat{n}_{m} \cdot\left(\vec{x}_{l}-\vec{x}_{m}\right) / R_{l m}, \quad l \neq m,
$$

while $N_{l m}^{\prime}, l \neq m$, is obtained from (46) by replacing $\hat{n}_{m}$ by $\hat{n}_{l}$. To obtain the self-patch contribution we expand the Hankel function, $\vec{x}^{\prime}$, and $\hat{n}^{\prime}$ to find

$$
N_{l l}=-N_{l l}^{\prime}=\frac{1}{4 \pi} \kappa_{l} \Delta s_{l}
$$

The sign of $\kappa$ is determined by the choice of $\hat{t}$ and $\hat{n}$ and the relation $d \hat{t} / d s=\kappa \hat{n}$. The term in (45) that is proportional to $(\Delta s) \log (\Delta s)$ may not be negligible compared to the sum of the other terms, and the curvature $\kappa$ in (47) can be large, for instance, when a sharp edge is being replaced by an arc of small radius. For patches near the self-patch, the variation of the Hankel functions with respect to $R$ may not be negligible, in which case special formulas can be developed for these neighboring patches.

The argument of a functional may be an unknown boundary function, a known homogeneous field, or another functional. A composite functional reduces to the product of the corresponding matrices of coefficients. By matching the values of the expressions on both sides of the integral equations (25)(28) evaluated at the points $\vec{x}_{l}$ we obtain the system of algebraic linear equations for the $\eta_{l}$.

The number of patches has to be such that the boundary is well approximated, and there have to be at least the usual number of about six to ten per wavelength. We have done computations with scatterers the size of a fraction of the wavelength without difficulty, but scatterers that are large compared to the wavelength are not easily accommodated by this method due to the large number of patches required to cover the boundaries.

\section{B. Field Computations}

The scattered electric field in $V_{1}$ is obtained by integration from $U_{1}$ in (15), the one in $V_{3}$ from $U_{3}$ in (16), and the total field in $V_{2}$ is calculated from (22). If we are interested only in the far fields, we can use the large argument approximation of the Hankel functions and reduce the problem to an integration of $\eta$ with the appropriate phase function, as shown in (40). On the other hand, if we need to calculate the fields near the cylinder we have to take into account that the Hankel functions become large for a small argument. Consequently, the integral over the patch or patches close to the field point has a rapidly varying integrand and cannot be well approximated by a constant. We can decrease the size of the patches until they are small compared to the distance between the field point and the curve, but this unnecessarily increases the number of points where the boundary functions have to be computed. It is better to subdivide the patches after solving the integral equations. We interpolate the values of the boundary functions, which vary slowly over the original patch, and compute the Hankel 
function (or a small-argument approximation) for each small patch.

\section{Interior Resonances}

Numerical instabilities have been predicted and observed $[15],[16]$ in calculations of scattered fields at frequencies that correspond to resonances for the interior boundary value problem for the scatterer. We have not encountered any of these problems in computations of fields scattered by a trapezoidal strip or a circular cylinder.

For the strip on a substrate, we have set the values of $\epsilon_{1}$ and $\epsilon_{3}$ equal to each other and chosen the dimensions of the strip so that the cross section is a square. The computation of the wave scattered by this square cylinder in free space did not show any problems even when the wavelength was set equal to the side of the square. A possible explanation for the absence of problems with internal resonances is the choice of boundary functions so that no single function covers the boundary of the cylinder.

\section{Effects of Sharp Edges}

Some components of the fields near the edge of a perfectly conducting or dielectric wedge have been determined to diverge with a power law that depends on the angle of the wedge [17]-[20], although there are doubts about the existence of the power series expansion used in the case of the dielectric. Locally, the same type of behavior is expected near any sharp edge.

The cylinder itself can have sharp edges, as in the example of the trapezoidal strip on the substrate, but even for a smooth cylinder the semi-infinite media have sharp edges where they meet the cylinder.

We use two different approaches to this problem in numerical calculations. Either we replace the sharp edge by a curve matched to the sides or we stay away from the edge itself in our selection of points.

The computed fields near the top surface depend strongly on the nearby values of the boundary functions. This dependence is enhanced because the Hankel functions diverge when the argument vanishes, but the more accurate integration over the patch does not help much near a sharp edge, especially since the interpolation is inaccurate for large oscillating values of the boundary functions.

\section{E. Results and Discussion}

We have written a group of computer programs to solve the problems of scattering by strips and cylinders of different media and configurations. We can calculate the intensity of the fields in the radiation zone as a function of angle, and this intensity is proportional to the radar cross section. We can also determine the fields anywhere and, for instance, the $y$ component of the Poynting vector near the scatterer.

In Fig. 2 we show the graphs of radar cross sections as a function of angle computed for a circular cylinder at the interface between two media. The first two graphs show results for a cylinder in a homogeneous medium for two

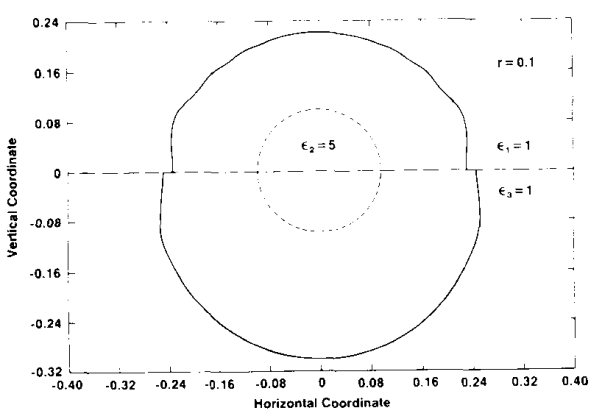

(a)

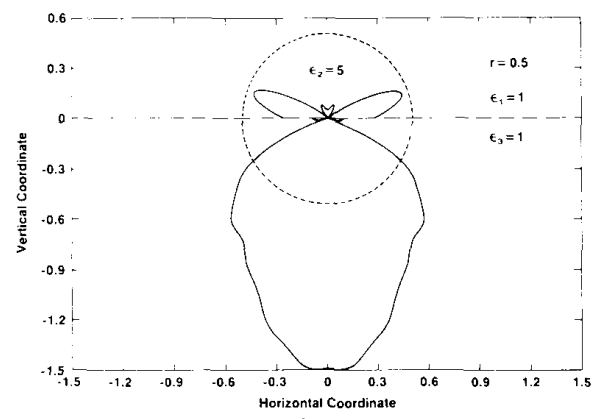

(b)

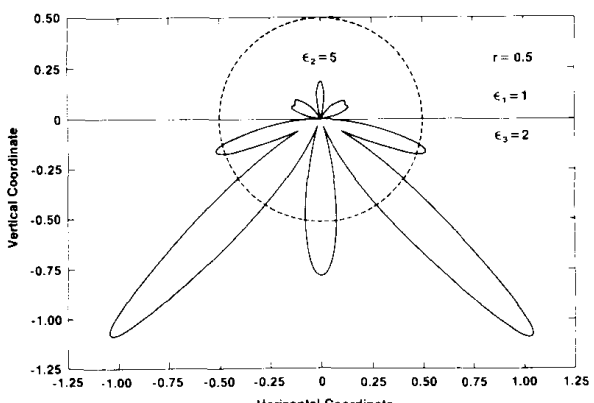

(c)

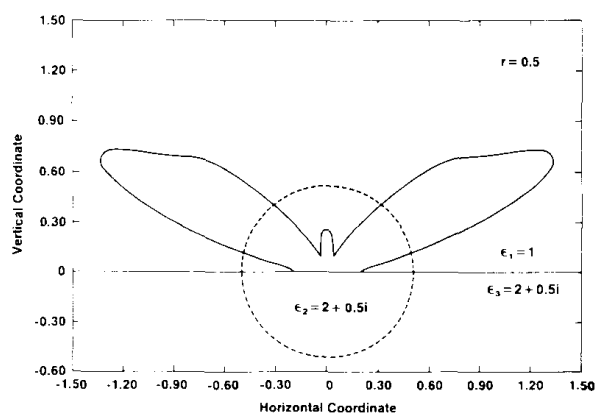

(d)

Fig. 2. Radar cross section of a circular cylinder. The radius, in multiples of the wavelength, and the permittivity are shown on the figures. All directions of incidence are vertical. The first two graphs correspond to cylinders of different radii in a homogeneous medium; (a) and (b) are obtained by setting $\epsilon_{1}=\epsilon_{3}$ in the general problem. In (c) the dielectric constants are real and $\epsilon_{1} \neq \epsilon_{3}$, and in (d) $\epsilon_{2}$ and $\epsilon_{3}$ are complex. 



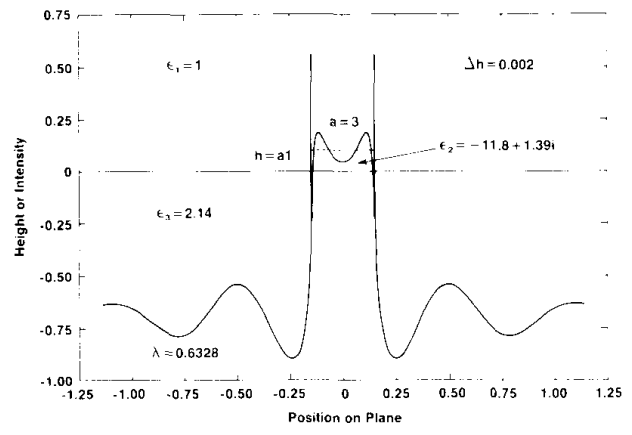

(a)

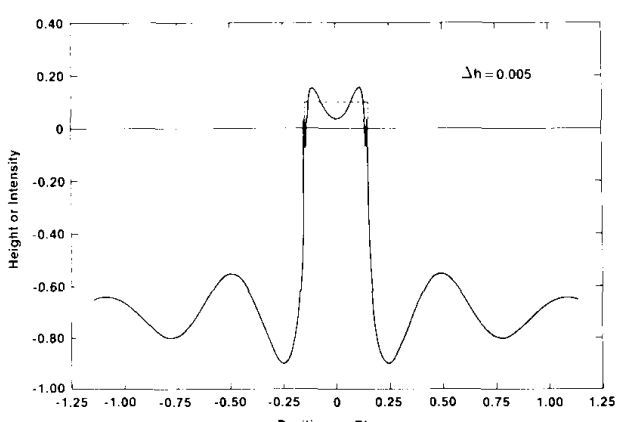

(b)

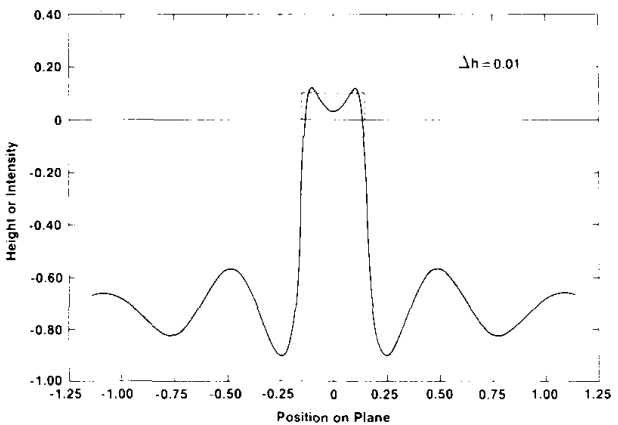

(c)

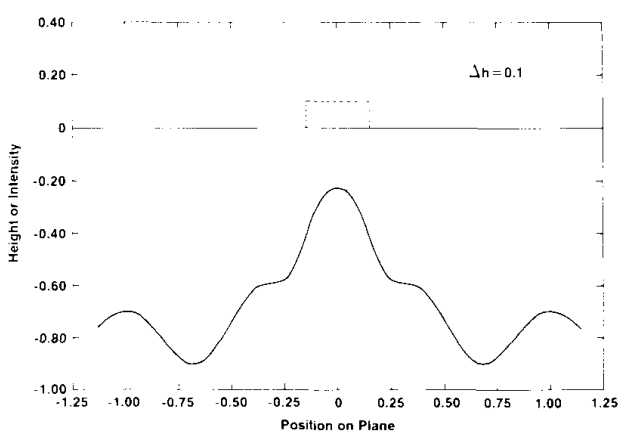

(d)

Fig. 4. Vertical component of the Poynting vector above a silver strip on a glass substrate for varying height above the strip. The dimensions are in micrometers. choose one that is discontinuous but has a continuous normal derivative.

The theory is exact, well suited to the resonance region, where the wavelength is comparable to the size of the scatterer. It is also applicable when the size of the scatterer is a fraction of the wavelength, but the number of unknowns grows significantly when the scatterer becomes large compared to the wavelength.

We can compute the scattered fields at an arbitrary location or we can use an approximation to compute the far fields as a function of scattering angles or radar cross sections. In this paper we have restricted ourselves to broadside incidence in the TE or TM modes. We have assumed that we have different dielectrics in the three regions, and we have allowed the cylinder to be perfectly conducting.

Similar equations can be derived for other configurations that have translational invariance and the same method can be adapted to compute scattered fields and radar cross sections of three-dimensional scatterers.

We have briefly described the process of implementation of these calculations on computers, and we have shown sample results from these calculations.

\section{ACKNOWLEDGMENT}

I thank Dr. Daniel Maystre, Dr. Ralph E. Kleinman, Dr. A. George Lieberman, Dr. David Gilsinn, and Dr. Jay F. Marchiando for helpful comments and suggestions.

\section{REFERENCES}

[1] D. Maystre, "Integral methods," in Electromagnetic Theory of Gratings, R. Petit. Ed. Berlin: Springer Verlag, 1980, ch. 3.

12] . "Electromagnetic scattering from perfectly conducting rough surfaces in the resonance region." IEEE Trans. Antennas Propagat. vol. AP-31, pp. 885-895, 1983

[3] E. Marx, "Single integral equation for wave scattering," J. Math. Phys. vol. 23, pp. 1057-1065, 1982.

[4] - "Integral equation for seattering by a dielectric." IEEE Trans. Antennas Propagat., vol. AP-32, pp. 166-172, 1984.

[5] . "Transient fields in dispersive media," J. Math. Phys., vol. 24. pp. $2602-2607,1983$.

[6] A. W. Glisson. "An integral equation for electromagnetic scattering from homogeneous dielectric bodies," IEEE Trans. Antennas Propagat., vol. AP-32, pp. 173-175, 1984.

[7] E. Marx, "Electromagnetic pulse seattered by a sphere." IEEE Trans. Antennas Propagat., vol. AP-35, pp. 412-417, 1987.

[8] - - , "Electromagnetic scattering by a thick strip on a half-space," Nat. Bur. Stand. (U.S.) Tech. Note 1236. 1987.

[9] _-_.., "Fields scattered by a dielectric strip on a dielectric half-space," in 1987 IEEE Antennas Propagat. Soc. Int. Symp. Dig., pp. $1138-$ 1141.

[10] E. Marx and E. C. Teague, "Determination of fields near a silver strip on a glass substrate," in SPIE Proc. Scanning Microscopy Technol. Appl.. vol. 897, 1988, pp. 176-184.

[11] C. M. Butler. "Current induced on a conducting strip which resides on the planar interface between to semi-infinite half-spaces." IEEE Trans. Antennas Propagat., vol. AP-34. pp. 226-231. 1984.

[12] E. Marx. "Scattering by an arbitrary cylinder at a plane interface," in Radar Cross Sections of Complex Objects, W. R. Stone, Ed. New York, IEEE Press, in press.

[13] K. M. Siegel, J. W. Crispin, and R. J. Newman, "RCS calculations of simple shapes-bistatic." in Methods of Radar Cross-Section Analysis, J. W. Crispin and K. M. Siegel. Eds. New York: Academic. 1968, p. 155

[14] J. J. Bowman, T. B. A. Senior, and P. L. E. Uslenghi, "Introduction," in Electromagnetic and Acoustic Scattering by Simple Shapes, J. J. 
Bowman. T. B. A. Senior, and P. L. E. Uslenghi, Eds. Amsterdam: North-Holland, 1969, p. 8

115] R. Mittra and C. R. Klein, "Stability and convergence of moment method solution," in Numerical and Asymptotic Techniques in Electromagnetics, R. Mittra, Ed. New York: Springer-Verlag, 1975, pp. $129-163$

[16] A. W. Glisson and B. I. Sholy, "Numerical solution of electromagnetic scattering problems involving homogeneous dielectric bodies via single integral equation," $1986 \mathrm{Nat}$. Radio Sci. Meet., p. 100.

[17] J. Meixner, "The behavior of electromagnetic fields at edges," IEEE Trans. Antennas Propagat., vol. AP-20, pp. 442-446, 1972

[18] J. Van Bladel, Electromagnetic Fields. New York: McGraw-Hill, 1964, p. 382.

[19] J. Bach Andersen and V. V. Solodukhov, "Field behavior near a dielectric wedge," IEEE Trans. Antennas Propagat., vol. AP-26, pp. 598-602, 1978

[20] E. Marx, "Edge effects on fields near a substrate," in Proc. 1988 URSI Radio Sci. Meet., p. 42.

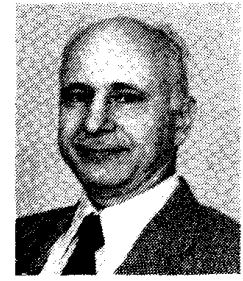

Egon Marx was born in Cologne. Germany, on April 4, 1937. He received the E.E. degree from the University of Chile, Santiago, Chile, in 1959, and the $\mathrm{Ph} . \mathrm{D}$. degree in physics from the California Institute of Technology, Pasadena, in 1963.

$\mathrm{He}$ has taught graduate and undergraduate courses in physics at the University of Chile. Clarkson College of Technology, and Drexel Uni versity, while doing research on the theory of classical and quantum fields and in relativistic quantum mechanics. In 1972 he joined the Harry Diamond Laboratories, where he worked on the effects of electromagnetic pulses and in the computer simulation of communications systems. Since 1980 he has been working at the National Institute of Standards and Technology (formerly the National Bureau of Standards) doing research on the theory and numerical applications of electromagnetic scattering, while also continuing with his work on the theory of elementary particles. 